\title{
A Hardware-In-The-Loop (HIL) Testbed for Hydraulic Transformers Research
}

\author{
Sangyoon Lee and Perry Y. Li \\ Department of Mechanical Engineering, University of Minnesota, Minneapolis, MN USA \\ E-mail: sylee@umn.edu, lixxx099@umn.edu
}

\begin{abstract}
A hardware-in-the-loop (HIL) testbed for testing and evaluating experimental hydraulic transformers has been developed. The testbed is capable of emulating a variety of loading conditions specified by the desired work cycle operation of various hydraulic machines, such as humanoid robots or excavators. The HIL testbed is a useful research tool as it allows studying the dynamics of many desired loading conditions without having to obtain and reconfigure the said physical hardware for each case. This paper discusses the background, construction, and control of the HIL testbed with the experimental results. Preliminary results demonstrating the functionality of an experimental switch mode hydraulic transformers are also presented.
\end{abstract}

Keywords: Hydraulic Transformer, Switch mode Transformer, Hardware-In-The-Loop testing, Load Emulation

\section{Introduction}

A hydraulic transformer transforms an input pressure and flow combination into an output pressure and flow combination in an energy conservative manner. It is a key component in realizing a common pressure rail (CPR) system where a common input pressure source feeds multiple services. Compared to valve based control, hydraulic transformers do not rely on throttling to transform pressures and allows energy regeneration. Thus they have the potential to improve hydraulic system efficiencies. Moreover, since pressure can be boosted as well as bucked, system pressure can be kept low to reduce losses.

Although much research has been done on hydraulic transformer configured using a rotatable 3-ported port plate such as the INNAS Hydraulic Transformer (IHT) in [1], a hydraulic transformer can also be configured simply as a combination of a hydraulic pump and motor connected mechanically on a common shaft. We refer to the latter as a Pump-motor or PM transformer. In a PM transformer, hydraulic power is converted by varying the relative displacements of the pump and of the motor. If the hydraulic transformer is configured with axial piston pump/motors, swash plate angles of the units will determine the pressure (or flow) transformation ratio.

In our previous work, we identified that 3 distinct configurations (PM-1, PM-2, PM-3) can be obtained by connecting the ports of a PM transformer variously to the supply pressure line, the load, and the return line (Fig. 1). By switching between these modes, a switch mode transformer has benefits in compactness, efficiency and operating region $[2,3]$.

While methodologies to design a hydraulic transformer as a component in a hydraulic system are well studied [4,5], con- trol algorithms for hydraulic transformer had received less attention. Werndin and Palmberg [6,7], Ahn and Ho [8] are among the few who investigated control algorithms for a hydraulic transformer to control actuators. In order to fill this gap, our group designed a passivity based backstepping controller and experimentally demonstrated the control performance for a trajectory tracking control [9] and a human power amplifier control $[10,11]$. These results were obtained for each PM transformer configuration, and suggest using the more efficient transformers need not sacrifice control performance as compared to control valves.

A major challenge in experimentally validating the control performance and efficiency of transformers in various applications and duty cycles is the difficulty of obtaining the needed hardware. It is often cost prohibitive to develop a machine and the loads. Consequently, a controller could only be tested for limited cases. Additionally, prototype transformers need to be duplicated to test a multi degree-of-freedom system.

A Hardware-In-the-Loop (HIL) testbed can provide a convenient solution for the aforementioned challenges. In a HIL system, the actual machine is replaced with a dynamic simulation executed in software, while the hydraulic component to be tested are presented physically with the pressure and flow conditions as in the actual machine. This allows a hydraulic component to be tested for a variety of operating scenarios without having to physically obtain the hardware.

Several HIL testbeds had been constructed by various research groups to overcome similar challenges in investigating hydraulic system. For example, Zhang et al [12] developed a HIL testbed to develop a controller for a hydraulic earthmoving vehicle, addressing the challenge in reproducing the digging cycles. Du et al [13] developed a hydrostatic dy- 


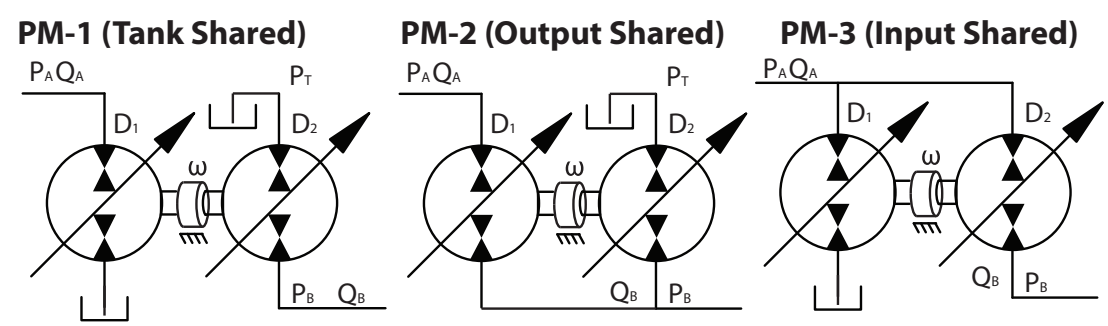

Figure 1: Three distinct transformer configurations

namometer for testing hydraulic hybrid vehicles, capable of emulating a variety of driving conditions and vehicle characteristics.

In this paper, a HIL testbed for hydraulic transformer is developed to test the performances of hydraulic transformer controlled systems. Each degree of freedom can be tested individually without needing the physical actuator or inertial load. The same testbed can also be used to test the transformer in different machine configurations and duty cycles by simply reprogramming the dynamic simulation. This testbed has a simple construction and can emulate both resistive and overrunning loads.

The rest of the paper is organized as follows. Section 2 describes the hydraulic transformer being studied in our group. Section 3 describes the HIL testbed construction and its control strategies. Implementation results and concluding remarks are given in Section 4 and 5.

\section{Hydraulic Transformer in Research}

The hydraulic transformer being studied in this paper is a traditional pump/motor type (PM transformer) configured with $3.15 \mathrm{cc} / \mathrm{rev}$ variable displacement micro-axial piston pump/motors from Takako. Three distinct configurations (PM-1, PM-2, PM-3) can be obtained by connecting the ports of a PM transformer differently (Fig. 1).

For each of the configuration, the transformer shaft speed $\omega$ acting with a rotational inertia $J$ to produce port flows out of transformer at input port $Q_{A}$ and output port $Q_{B}$ are given by following sets of equations:

PM-1:

$$
\begin{aligned}
J \dot{\omega} & =\left(P_{A}-P_{T}\right) \frac{D_{1}}{2 \pi} u_{1}+\left(P_{T}-P_{B}\right) \frac{D_{2}}{2 \pi} u_{2}-b_{t} \omega-T_{\text {loss }} \\
Q_{A} & =-\omega \cdot \frac{D_{1}}{2 \pi} u_{1}-Q_{\text {leak }^{\prime}} \\
Q_{B} & =\omega \cdot \frac{D_{2}}{2 \pi} u_{2}-Q_{\text {leak }}
\end{aligned}
$$

PM-2:

$$
\begin{aligned}
J \dot{\omega} & =\left(P_{A}-P_{B}\right) \frac{D_{1}}{2 \pi} u_{1}+\left(P_{T}-P_{B}\right) \frac{D_{2}}{2 \pi} u_{2}-b_{t} \omega-T_{\text {loss }} \\
Q_{A} & =-\omega \cdot \frac{D_{1}}{2 \pi} u_{1}-Q_{\text {leak }} \\
Q_{B} & =\omega \cdot\left(\frac{D_{1}}{2 \pi} u_{1}+\frac{D_{2}}{2 \pi} u_{2}\right)-Q_{\text {leak }}
\end{aligned}
$$

PM-3:

$$
\begin{aligned}
J \dot{\omega} & =\left(P_{A}-P_{T}\right) \frac{D_{1}}{2 \pi} u_{1}+\left(P_{A}-P_{B}\right) \frac{D_{2}}{2 \pi} u_{2}-b_{t} \omega-T_{\text {loss }} \\
Q_{A} & =-\omega \cdot\left(\frac{D_{1}}{2 \pi} u_{1}+\frac{D_{2}}{2 \pi} u_{2}\right)+Q_{\text {leak }} \\
Q_{B} & =\omega \cdot \frac{D_{2}}{2 \pi} u_{2}-Q_{\text {leak }}
\end{aligned}
$$

where $D_{1}$ and $D_{2}$ are the maximum volumetric displacements of the pump/motor units in $\mathrm{m}^{3} / \mathrm{rev}, u_{1}$ and $u_{2} \in[-1,1]$ are the control inputs which are the normalized displacements, $b_{t}$ is the damping coefficient, $Q_{\text {leak }}, Q_{\text {leak }}$ and $T_{\text {loss }}$ are the lumped volumetric losses at the $\mathrm{A}$ and $\mathrm{B}$ ports and the mechanical loss inside the transformer due to friction. These losses are configuration, pressure and shaft speed dependent.

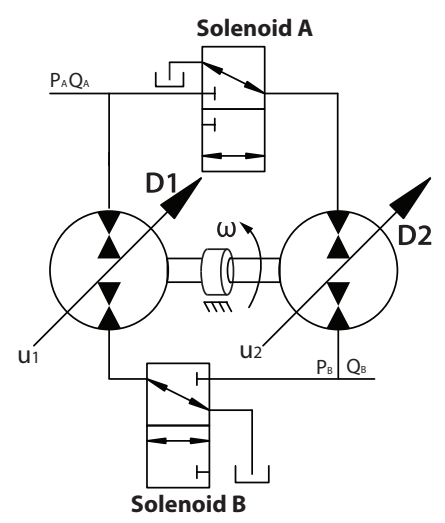

Figure 2: Switch Mode Transformer

Adding two 3-way solenoid valves as in Fig. 2 allows all three configurations to be realized. By switching between the configurations, the total volumetric displacement of the pump/motors (hence overall size) can be reduced for a given flow capability and shaft speed [2,3]. For example, a switched mode PM transformer needs only be $13 \%$ larger than IHT with equivalent flow capability as compare to $33 \%$ larger when compared to using just one configuration. In addition, by picking the configuration with the highest efficiency, the overall transformer efficiency and operating region can be improved.

Fig. 3 shows the efficiency map of an early stage prototype transformer with a constant $6.895 \mathrm{MPa}$ (1000 psi) pressure rail for each configuration. The relatively low efficiency of this prototype is due primarily to the unusually high mechan- 


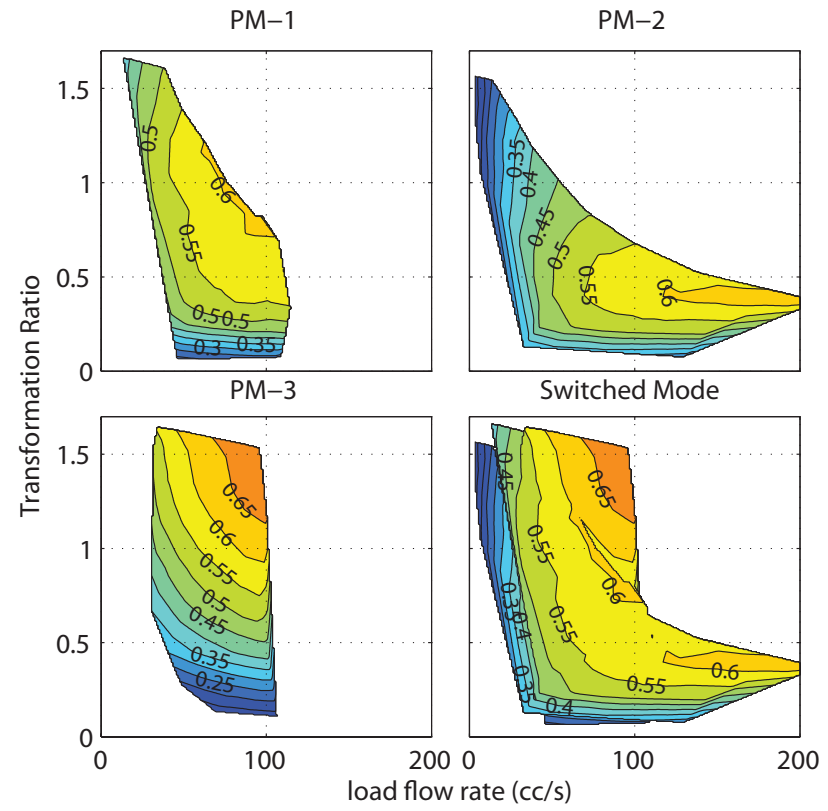

Figure 3: Efficiency contours of individual PM-1, PM-2, PM3 transformer configurations and switched configurations

ical loss of the common shaft. Incorporating a bearing is expected to significantly improve the efficiency. Nevertheless, this prototype can still demonstrate the implications of using a hydraulic transformer for system control. One can also discern that the switch mode transformer widens the operating region with high efficiency by picking the best configuration for each operating point (Fig. 3, bottom right).

In our prototype switch mode transformer, two Sun Hydraulics DMDA-MBN 3-way solenoid-operated directional spool valves are placed at both sides of the transformer as shown in Fig. 2. At the default position, the transformer is configured in 'PM-1' configuration, which has the shared line connected to the return line. If solenoid valve B is triggered, then the transformer is configured in 'PM-2' configuration, with the shared output port. Lastly, if solenoid A is triggered, the transformer is in 'PM-3' configuration with the input port shared between two units.

\section{HIL Testbed for Hydraulic Transformer}

\subsection{Components of HIL System Architecture}

The hydraulic schematic of the HIL Testbed for hydraulic transformer is shown in Fig. 4. The system is powered by a main pressure compensated pump, which provides, via a pressure reducing valve, the constant pressure $P_{S}$ to the system.

Switch mode transformer discussed in Section 2 is being studied with this HIL Testbed. As mentioned, actuating the solenoid valves switches the hydraulic transformer to be in one of the three configurations shown in Fig. 1. This setup can also be used to study other transformer designs, such as an IHT.

Pressure $(P)$ and flow $(Q)$ sensors are placed at various places as shown in Fig. 4. The transformer shaft speed $\omega$ is meas-

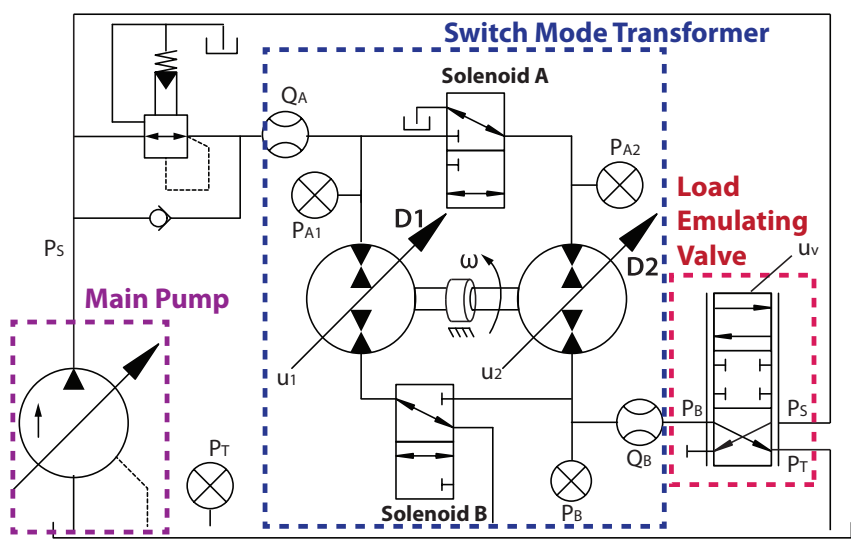

Figure 4: HIL Circuit for testing Switch Mode Transformer

ured with an optical encoder. The transformer controller determines the displacement control inputs $u_{1}$ and $u_{2}$ that will deliver the desired flow $Q_{B}^{d}$ to the actuator while maintaining the desired shaft speed $\omega_{d}[9,10]$.

In the HIL testbed, a load emulating valve (LEV) is utilized in place of the hydraulic actuator. A Moog 760 series servo valve (rated at 9.5 LPM), connected to the main pump prior to the pressure reducing valve and to tank, is used for this purpose. One outlet port is connected to the transformer output while the other port is blocked. As will be described in Section 3.4, the servo valve command $u_{v}$ is used to create an appropriate pressure condition for the transformer that mimics the actual load condition.

The fact that the LEV is connected to a supply pressure $P_{S}$ is higher than the input port pressure of the transformer $P_{A}$ allows this HIL system to emulate both resistive and overrunning loads just using one main pump. This configuration is advantageous as only one main pump is needed to power the whole testbed as opposed to having separate pumps for emulating load and running a hydraulic transformer.

\subsection{Data and Signal Flow in the HIL Testbed}

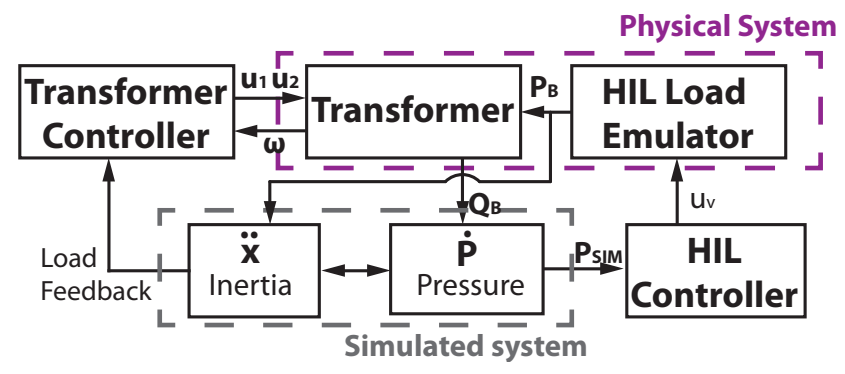

Figure 5: HIL Control Scheme

Fig. 5 shows the block diagram illustrating the data flow within the testbed. The output flow $Q_{B}$ from the transformer is measured and is provided to simulate the pressure dynamics $\dot{P}$, creating a simulated pressure $P_{S I M}$ in return. In case of a hydraulic actuator shown in Fig. 6, the pressure dynamics of the capside chamber which is to be (virtually) connected to 


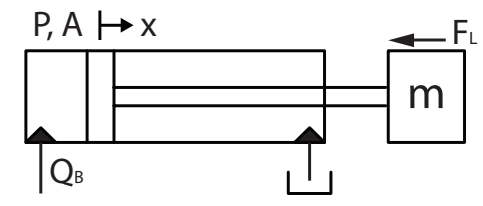

Figure 6: Example cylinder being simulated

the transformer is given by:

$$
\dot{P}_{S I M}=\frac{\beta}{V_{0}+A x}\left(Q_{B}-A \dot{x}\right)
$$

where $V_{0}$ is the volume in the capside chamber and hose when the actuator is at the position $x=0$, and $\beta$ is the bulk modulus of the fluid. Integrating $\dot{P}_{S I M}$ yields a $P_{S I M}$ which is fed into a HIL controller to determine the LEV control $u_{v}$ to match the actual pressure $P_{B}$ with $P_{S I M}$.

The measured pressure $P_{B}$ in turn drives the simulated inertia dynamics, $\ddot{x}$ :

$$
m \ddot{x}=-b \dot{x}+\underbrace{P_{B}(t)}_{\text {measured }} A+F_{L}
$$

where $m$ is the mass of the cylinder and rod to be simulated, $A$ is the cap side area of the hydraulic actuator, $b$ is the viscous friction coefficient, and $F_{L}$ is the load force that would encapsulate any external load including gravity and environment forces. Integrating $\ddot{x}$ yields the velocity $\dot{x}$ and the position $x$ which are used in (4). Note that this simulated inertia dynamics take the measured pressure to account for any movement change due to pressure dynamics associated with transformer mode switch.

Finally, the transformer controller takes the feedback of the above information to determine the transformer control inputs $u_{1}$ and $u_{2}$ to achieve the desired flow for the (emulated) load and the desired torque for the (actual) transformer speed regulation.

The pressure dynamics $\dot{P}$ and the inertia dynamics $\ddot{x}$ in (4)-(5) can be set to simulate any desired actuator type with specified loading conditions, generating appropriate motion and mechanical load. If desired, the simulated system dynamics could simply be replaced by a duty cycle information providing the desired pressure and flow traces.

\subsection{Hydraulic Transformer Controller}

The objective of the hydraulic transformer controller is to provide the desired flow $Q_{B}^{d}$ for the given task and the desired torque $T^{d}$ to regulate the shaft speed $[9,10]$.

PM-1:

$$
\left[\begin{array}{l}
u_{1} \\
u_{2}
\end{array}\right]=\left[\begin{array}{cc}
0 & \omega \cdot \frac{D_{2}}{2 \pi} \\
\left(P_{A}-P_{T}\right) \frac{D_{1}}{2 \pi} & \left(P_{T}-P_{B}\right) \frac{D_{2}}{2 \pi}
\end{array}\right]^{-1}\left[\begin{array}{l}
Q_{B}^{d} \\
T^{d}
\end{array}\right]
$$

PM-2:

$$
\left[\begin{array}{l}
u_{1} \\
u_{2}
\end{array}\right]=\left[\begin{array}{cc}
\omega \cdot \frac{D_{1}}{2 \pi} & \omega \cdot \frac{D_{2}}{2 \pi} \\
\left(P_{A}-P_{B}\right) \frac{D_{1}}{2 \pi} & \left(P_{T}-P_{B}\right) \frac{D_{2}}{2 \pi}
\end{array}\right]^{-1}\left[\begin{array}{l}
Q_{B}^{d} \\
T^{d}
\end{array}\right]
$$

PM-3:

$$
\left[\begin{array}{l}
u_{1} \\
u_{2}
\end{array}\right]=\left[\begin{array}{cc}
0 & \omega \cdot \frac{D_{2}}{2 \pi} \\
\left(P_{A}-P_{T}\right) \frac{D_{1}}{2 \pi} & \left(P_{A}-P_{B}\right) \frac{D_{2}}{2 \pi}
\end{array}\right]^{-1}\left[\begin{array}{l}
Q_{B}^{d} \\
T^{d}
\end{array}\right]
$$

\subsection{Load Emulating Valve (LEV) Controller}

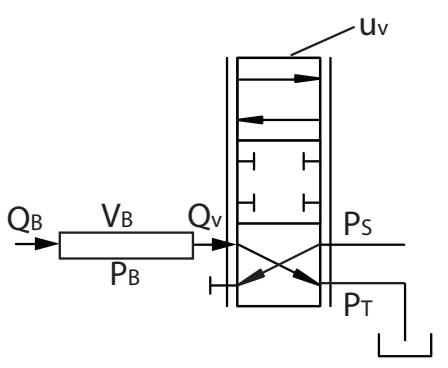

Figure 7: Load Emulating Valve

The most important aspect of the HIL testbed is the load emulating valve (LEV) reproduced in Fig. 7, which will provide the desired loading condition through pressure. For $u_{v}>0$, the flow is released to the tank (as shown on the figure) and for $u_{v}<0$, the flow is taken from the main pump to increase pressure. The flow $Q_{v}$ traveling across the valve from the transformer output to be exposed to $P_{T}$ or $P_{S}$ is given by the supply pressure or the tank pressure:

$$
Q_{v}= \begin{cases}k_{v} u_{v} \sqrt{\left|P_{B}-P_{T}\right|}, & \text { for } u_{v}>0 \\ k_{v} u_{v} \sqrt{\left|P_{B}-P_{S}\right|}, & \text { for } u_{v}<0\end{cases}
$$

(assuming $P_{S}>P_{B}>P_{T}$ ) where $k_{v}$ is the valve coefficient, and $u_{v}$ is the valve command to be designed. For the controller design, consider the pressure dynamics within the hose that lies between the hydraulic transformer and the LEV:

$$
\dot{P}_{B}=\frac{\beta}{V_{B}}\left(Q_{B}-Q_{v}\right)
$$

where $V_{B}$ is the volume of the hose between the transformer and the LEV, $Q_{B}$ is the flow out of the transformer that is measured, and $Q_{v}$ is the flow command to be designed. Define pressure error as $e=P_{B}-P_{S I M}$. For a constant (or slowly changing) desired simulated pressure, $\dot{e}=\dot{P}_{B}$. Defining the desired valve port flow $Q_{v}^{d}$ as:

$$
Q_{v}^{d}=Q_{B}+K_{p} e+K_{I} e_{I}
$$

where $\dot{e}_{I}=e$ and $K_{p}$ and $K_{I}$ are the proportional and integral gains. To show that the desired pressure $P_{S I M}$ can be achieved for the HIL testbed, consider the Lyapunov function:

$$
\begin{aligned}
& W=\frac{1}{2} \frac{V_{B}}{\beta} e^{2}+\frac{1}{2} K_{I} e_{I}^{2} \\
& \dot{W}=e\left(Q_{B}-Q_{v}\right)+e K_{I} e_{I}
\end{aligned}
$$

With $Q_{v}$ given by (11),

$$
\dot{W}=-K_{p} e^{2}
$$

which shows that for $K_{p}>0, K_{I}>0, e \rightarrow 0$ and $P_{B}$ will track $P_{S I M}$, providing the simulated pressure.

In summary, the LEV controller is a PI controller with a feedforward information coming from the measured flow out the transformer. 


\section{Experimental Results}

\subsection{Pressure Tracking Results}

To validate that (11) is appropriate, the transformer displacements were held steady while only the desired pressure loading condition was varied. This type of experiment can be used to analyze the steady-state operation for the hydraulic transformer under various loading conditions.

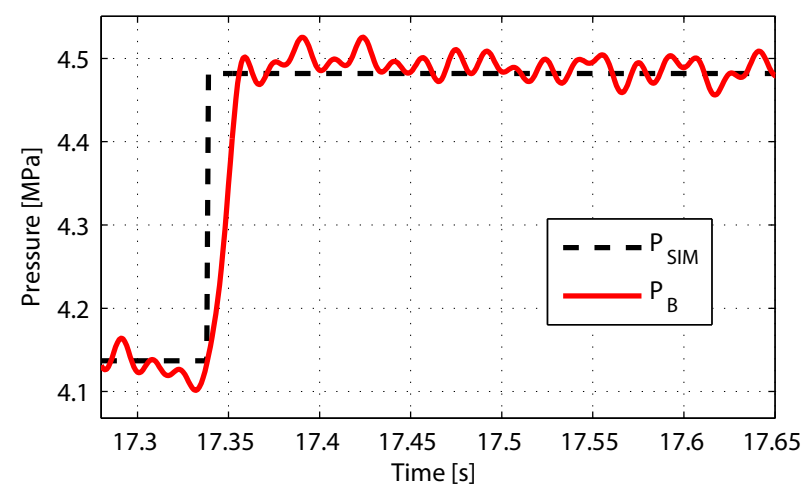

Figure 8: Step response

Fig. 8 shows the step pressure response for a $0.345 \mathrm{MPa}(50$ psi) step pressure command. Negligible overshoot with $10 \mathrm{~ms}$ rise time is observed. Fig. 9 shows various responses of the same step size.

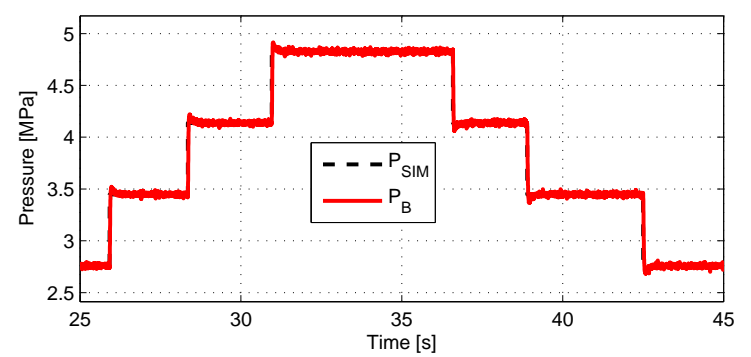

Figure 9: Various step responses

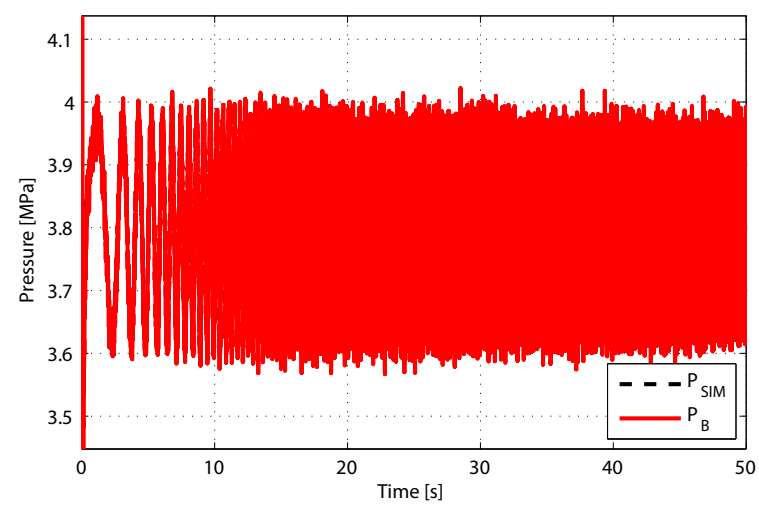

Figure 10: Chirp signal response

Figure 10 shows a response for the chirp signal and Fig. 10 shows the same response zoomed around at select frequen-
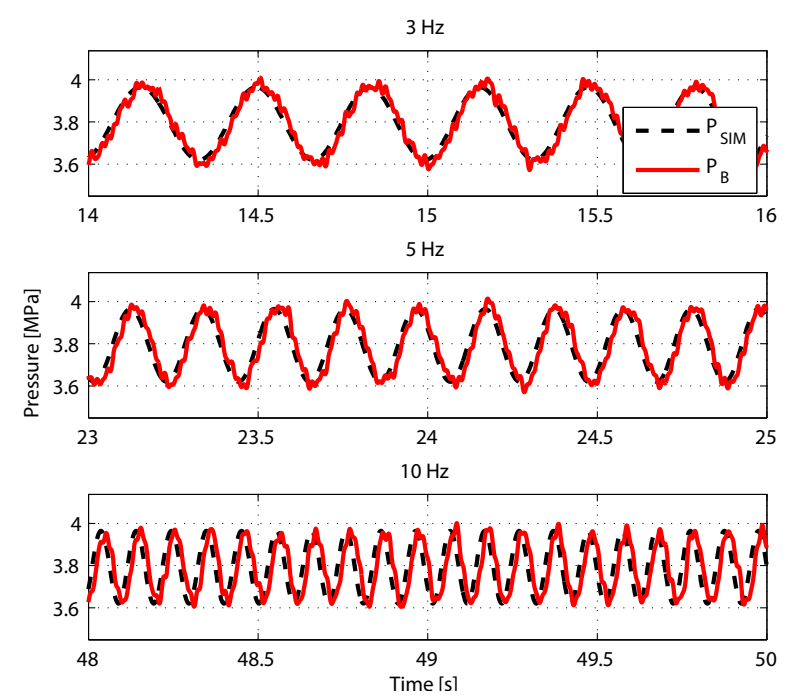

Figure 11: Chirp signal zoomed in at select frequencies

cies. The reference chirp signal, an amplitude of $0.345 \mathrm{MPa}$ (50 psi), started as $0.01 \mathrm{~Hz}$ at $t=0$ and approached $10 \mathrm{~Hz}$ at $t=50$. Phase lag of 36 degree was observed at the peak frequency, while phase lag is negligible around $3 \mathrm{~Hz}$. Thus, it will be safe to claim this testbed can be operated satisfactorily under $3 \mathrm{~Hz}$ load profile. Careful tuning of the parameters along with better valve identification will lead to even better performance. As transformer itself has a limited bandwidth due to the swashplate actuation, HIL testbed is more than capable of simulating the loads for the transformer.
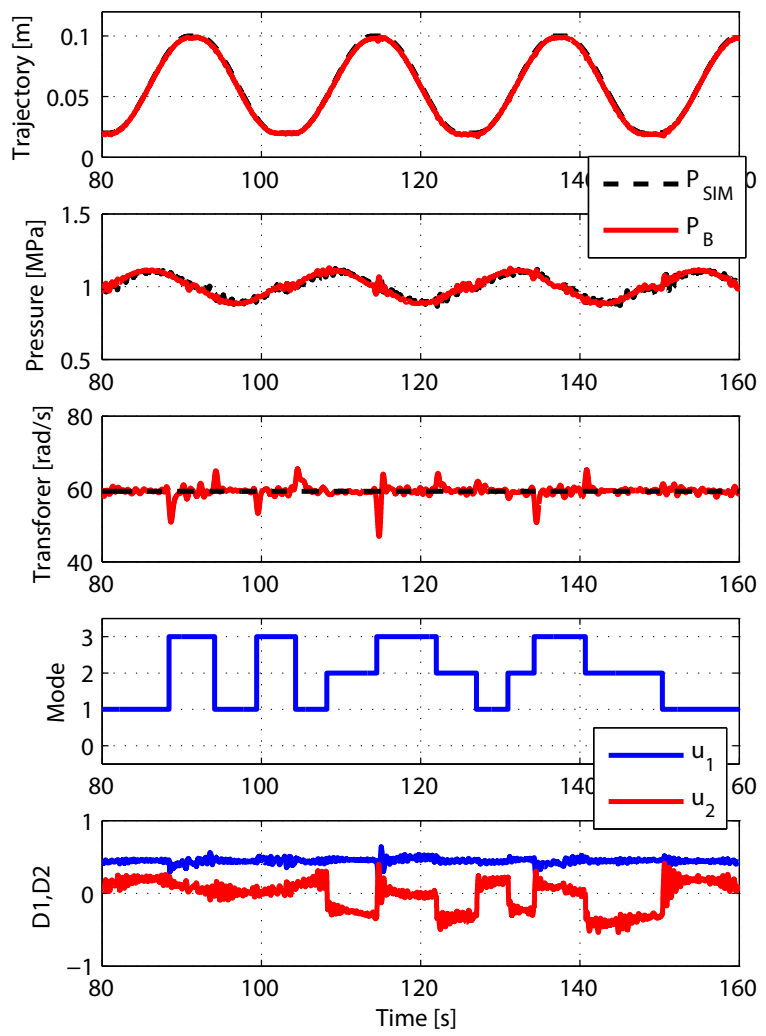

Figure 12: Simulated cylinder tracking result 


\subsection{Flow and Pressure Tracking Results}

An operating condition where a LEV and transformer are simultaneously controlled is shown in Fig. 12. For this implementation, a cylinder with vertical gravity load following a filtered trapezoidal trajectory was studied with a transformer operating in 3 different modes. Controller determining flow demand and the transformer displacement commands is taken from [9]. While the transformer mode switch is manually triggered for this experiment, further development of the transformer control will have the mode automatically determined to maximize the operating efficiency.

In this operating mode, HIL provides a loading condition to which trajectory tracking controller decides the transformer control inputs to be implemented. As described, the trajectory is generated in simulation using the measured flow and pressure. It is observed that trajectory tracking is still satisfactory even as the operating mode switch causes some sharp change of dynamics. In the meantime, HIL control is tracking the simulated pressure $P_{S I M}$.

\section{Conclusion}

This paper presents a development of a HIL testbed for hydraulic transformer, with a focus on the control architecture. Using a single hydraulic power supply and a servo-valve, a HIL testbed is configured, providing a platform for further transformer control development.

A closed loop controller designed for the actual system for a trajectory tracking is easily implemented for the HIL testbed. Initial performance experiments indicates the ability of this testbed to emulate various loading conditions.

\section{Acknowledgment}

This work is performed within the Center for Compact and Efficient Fluid Power (CCEFP) supported by the National Science Foundation under grant EEC-05040834. Component donation from Takako Industries is gratefully acknowledged. Sangyoon Lee is supported by a 2016-2017 Doctoral Dissertation Fellowship at the University of Minnesota.

\section{Nomenclature}

\begin{tabular}{|c|c|c|}
\hline Designation & Denotation & Unit \\
\hline$A$ & Piston Area & $\mathrm{m}^{2}$ \\
\hline$b$ & $\begin{array}{l}\text { Cylinder viscous friction coeffi- } \\
\text { cient }\end{array}$ & $\mathrm{N} /(\mathrm{m} / \mathrm{s})$ \\
\hline$b_{t}$ & $\begin{array}{l}\text { Transformer viscous friction } \\
\text { coefficient }\end{array}$ & $\mathrm{N} \cdot \mathrm{m} \cdot \mathrm{s}$ \\
\hline$D_{1}, D_{2}$ & Volumetric displacements & $\mathrm{m}^{3} / \mathrm{rev}$ \\
\hline$e$ & Pressure error & $\mathrm{Pa}$ \\
\hline$e_{I}$ & Integral of pressure error & $\mathrm{Pa}$ \\
\hline$F_{L}$ & Load force & $\mathrm{N}$ \\
\hline$J$ & Transformer shaft inertia & $\mathrm{kg} \cdot \mathrm{m}^{2}$ \\
\hline$K_{I}$ & Integral control Gain & \\
\hline$K_{p}$ & Proportional control gain & \\
\hline$k_{v}$ & LEV valve coefficient & \\
\hline$m$ & Mass of cylinder & $\mathrm{kg}$ \\
\hline$P_{A}, P_{B}, P_{T}$ & Transformer port pressures & $\mathrm{Pa}$ \\
\hline$P_{S}$ & Main pump supply pressure & $\mathrm{Pa}$ \\
\hline$P_{S I M}$ & Simulated pressure & $\mathrm{Pa}$ \\
\hline$Q_{A}, Q_{B}$ & Transformer flows & $\mathrm{m}^{3} / \mathrm{s}$ \\
\hline$Q_{B}^{d}$ & Desired flow of transformer & $\mathrm{m}^{3} / \mathrm{s}$ \\
\hline$Q_{\text {leak }}, Q_{\text {leak' }}$ & Flow loss of transformer & $\mathrm{m}^{3} / \mathrm{s}$ \\
\hline$Q_{v}$ & Flow across the valve & $\mathrm{m}^{3} / \mathrm{s}$ \\
\hline$Q_{v}^{d}$ & Desired flow across the valve & $\mathrm{m}^{3} / \mathrm{s}$ \\
\hline$T^{d}$ & Desired torque for transformer & $\mathrm{N} \cdot \mathrm{m}$ \\
\hline$T_{\text {loss }}$ & Mechanical loss of transformer & $\mathrm{N} \cdot \mathrm{m}$ \\
\hline$u_{1}, u_{2}$ & Displacement ratio inputs & \\
\hline$u_{v}$ & Valve command & \\
\hline$V_{0}$ & $\begin{array}{l}\text { Initial volume in cylinder cap- } \\
\text { side }\end{array}$ & $\mathrm{m}^{3}$ \\
\hline$V_{B}$ & Fluid volume in hose & $\mathrm{m}^{3}$ \\
\hline$W$ & Lyapunov energy & $\mathbf{J}$ \\
\hline$x$ & Piston displacement & $\mathrm{m}$ \\
\hline$\beta$ & Bulk modulus & $\mathrm{Pa}$ \\
\hline$\Delta P_{v}$ & Pressure drop across the valve & $\mathrm{Pa}$ \\
\hline$\omega$ & Transformer shaft speed & $\mathrm{rad} / \mathrm{s}$ \\
\hline
\end{tabular}

\section{References}

[1] P.A.J. Achten, Z. Fu, and G.E.M. Vael. Transforming future hydraulics: a new design of a hydraulic transformer. In The Fifth Scandinavian International Conference on Fluid Power SICFP '97, page 287ev, 1997.

[2] Sangyoon Lee, Pieter Gagnon, and Perry Y Li. System configuration and control using hydraulic transformers. In Fluid Power Innovation and Research Conference, Chicago, IL, October. 14-16 2015.

[3] Pieter J Gagnon. Configuration and performance of hydraulic transformer power distribution systems. Master's thesis, University of Minnesota, 2016.

[4] Peter AJ Achten, Titus van den Brink, Johan van den Oever, Jeroen Potma, Marc Schellekens, Georges Vael, Martijn van Walwijk, and BV Innas. Dedicated design of the hydraulic transformer. volume 3, pages 233-248, 2002. 
[5] PAJ Achten, TL Van den Brink, T Paardenkooper, T Platzer, HW Potma, MPA Schellekens, and GEM Vael. Design and testing of an axial piston pump based on the floating cup principle. In The Eighth Scandinavian International Conference on Fluid Power SICFP '03, pages 805-820, May 2003.

[6] R. Werndin and J-O Palmberg. Controller design for a hydarulic transformer. In The Fifth International Conference on Fluid Power Transmission and Control ICFP '01, volume 5, pages 56-61, 2001.

[7] R. Werndin and J-O Palmberg. Hydraulic transformer in low-speed operation - a study of control strategies. In The 5th International Symposium of Fluid Power, JFPS '02, Nara, Japan, 2002.

[8] Triet Hung Ho and Kyoung Kwan Ahn. A study on the position control of hydraulic cylinder driven by hydraulic transformer using disturbance observer. In International Conference on Control, Automation and Systems 2008, pages 2634-2639. IEEE, 2008.

[9] Sangyoon Lee and Perry Y Li. Passivity based backstepping control for trajectory tracking using a hydraulic transformer. In ASME/BATH 2015 Symposium on Fluid Power and Motion Control, pages V001T01A064V001T01A064. American Society of Mechanical Engineers, 2015.

[10] Sangyoon Lee and Perry Y Li. Passive control of a hydraulic human power amplifier using a hydraulic transformer. In ASME 2015 Dynamic Systems and Control Conference, pages V002T27A004-V002T27A004. American Society of Mechanical Engineers, 2015.

[11] Sangyoon Lee, Fredrik Eskilsson, and Perry Y Li. Multi degree-of-freedom hydraulic human power amplifier with rendering of assistive dynamics. In $A S M E$ 2016 Dynamic Systems and Control Conference, page V001T07A002. American Society of Mechanical Engineers, 2016.

[12] Rong Zhang, Don E Carter, and Andrew G Alleyne. Multivariable control of an earthmoving vehicle powertrain experimentally validated in an emulated working cycle. In ASME 2003 International Mechanical Engineering Congress and Exposition, pages 515-524. American Society of Mechanical Engineers, 2003.

[13] Zhekang Du, Tan Cheng, Perry Y Li, Kai Loon Cheong, and Thomas R Chase. Virtual vehicle control concept for hydrostatic dynamometer control. Journal of Dynamic Systems, Measurement, and Control, 139(2):021009, 2017. 